\title{
Utilización de un sistema de destilación solar para remoción de Arsénico y Flúor en agua subterránea destinada al consumo humano
}

\section{Use of a solar distillation system for the removal of arsenic and fluorine in groundwater intended for human consumption}

ESPINO-VALDÉS, María Socorro ${ }^{*}$, HERNÁNDEZ-HERRERA, Cristina, PINALES-MUNGUÍA, Adán y BALLINAS-CASARRUBIAS, María de Lourdes

Universidad Autónoma de Chihuahua. Facultad de Ingeniería

ID $1^{\text {er }}$ Autor: María Socorro, Espino-Valdés / ORC ID: 0000-0002-1232-2774, CVU CONACYT ID: 202985

ID $1^{\text {er }}$ Coautor: Cristina, Hernández-Herrera / ORC ID: 0000-0003-3675-8385, CVU CONACYT ID: 768669

ID $2^{\text {do }}$ Coautor: Adán, Pinales-Munguía / ORC ID: 0000-0002-1921-7168, CVU CONACYT ID: 123798

ID $3^{\text {er }}$ Coautor: María de Lourdes, Ballinas-Casarrubias / ORC ID: 0000-0001-5510-645X, CVU-CONACYT ID: 120754

DOI: $10.35429 / J B E B .2019 .8 .3 .14 .22$

Recibido 16 de Marzo, 2019; Aceptado 30 Junio, 2019

\section{Resumen}

El arsénico y el flúor se presentan de manera natural en las aguas subterráneas, especialmente en las zonas desérticas y semidesérticas. La exposición crónica a dichas sustancias a través del agua de bebida provoca efectos adversos a la salud, por lo que sus concentraciones están limitadas en el agua destinada al consumo humano. Objetivo: Este trabajo se realizó con la finalidad de mejorar la calidad del agua procedente de pozos con alto contenido de arsénico y flúor, logrando el cumplimiento de los límites establecidos en la normatividad mexicana para agua potable. Metodología: Se utilizó un sistema de destilación solar de dos vertientes para el tratamiento del agua procedente de tres pozos caracterizados por sus altos niveles de arsénico y flúor, los cuales se destinan al consumo humano. Contribución : El sistema de destilación solar empleado mejoró la calidad del agua subterránea mediante la remoción de arsénico (99\%), flúor (96\%) y sólidos disueltos (98\%). El sistema probado es una alternativa viable para el tratamiento de agua en pequeñas comunidades que no tienen acceso a plantas desmineralizadoras de agua que operan con grandes costos, eliminando de este modo los riesgos de salud asociados a la ingesta crónica de dichos elementos.

Agua subterránea, Arsénico y Flúor, Destilación solar

\begin{abstract}
Arsenic and fluorine occur naturally in groundwater, especially in desert and semi-desert areas. Chronic exposure through drinking water to these substances causes adverse health effect, which are limited in water intended for human consumption. Objective: This work was carried out with the aim of improving the quality of water from wells with high contents of arsenic and fluorine, achieving compliance with the limits established in the Mexican regulations for drinking water. Methodology: A system of two-panel solar distillation was used for treatment of water from three wells characterized by high levels of arsenic and fluoride, which are intended for human consumption. Contribution: The solar distillation system used improved the quality of the groundwater through the removal of arsenic (99\%), fluoride $(96 \%)$ and dissolved solids $(98 \%)$. The proven system is a viable alternative for water treatment in small communities that do not have access to demineralization water plants that operate with high costs, thus eliminating health risks associated to the chronic intake of these elements.
\end{abstract}

Groundwater, Arsenic and Fluorine, Solar distillation

Citación: ESPINO-VALDÉS, María Socorro, HERNÁNDEZ-HERRERA, Cristina, PINALES-MUNGUÍA, Adán y BALLINAS-CASARRUBIAS, María de Lourdes. Utilización de un sistema de destilación solar para remoción de Arsénico y Flúor en agua subterránea destinada al consumo humano. Revista de Ingeniería Biomédica y Biotecnología. 2019. 3-8: 1422

\footnotetext{
* Correspondencia del Autor (msespinov27@gmail.com)

$\dagger$ Investigador contribuyendo como primer autor
} 


\section{Introducción}

A nivel mundial las aguas subterráneas constituyen la fuente principal de agua dulce; por lo menos el 50\% de la población depende exclusivamente de los recursos subterráneos para satisfacer sus necesidades básicas diarias de agua (CONAGUA, 2011). De acuerdo con el tipo de ambiente hidrogeológico en el que se encuentre, el agua subterránea presenta diferencias en calidad. Debido a su gran capacidad como solvente es capaz de incorporar de una gran variedad de sustancias; algunas de éstas, cuando se encuentran en grandes cantidades, pueden ocasionar daños en la salud de los consumidores. Entre dichos constituyentes se cuentan los fenoles, el arsénico, selenio, cianuros, cromo, plomo, mercurio, flúor, nitratos y compuestos orgánicos clorados, entre otros (CONAGUA, 2007).

El arsénico se presenta de forma natural en el medio ambiente tanto en aguas subterráneas como superficiales, al igual que en muchos alimentos. En las aguas naturales proviene principalmente de la deposición atmosférica, la disolución de minerales y la erosión de las rocas. Otra fuente posible de contaminación es el uso de plaguicidas en la agricultura (Castro De Esparza, 2006).

Entre las manifestaciones más comunes de la presencia del arsénico en el organismo se encuentran alteraciones en la piel que incluyen cambios de pigmentación, hiperqueratosis en manos y pies y otras lesiones cutáneas. Se considera que estos efectos pueden ser precursores del cáncer de piel (Bocanegra, 2002).

La exposición crónica aún a bajos niveles de arsénico ha sido vinculada también con cáncer de riñón y vejiga, así como otros problemas del sistema cardiovascular y enfermedades neurológicas (EPA, 2016). Al menos cuatro millones de personas en países de América Latina como Argentina, Chile, Nicaragua, El Salvador, Perú y México beben agua con niveles de arsénico que constituyen un riesgo para su salud (Castro de Esparza, 2006).

El flúor es otro de los elementos químicos que producen efectos significativos en los seres humanos a través del consumo de agua potable. Al ser ingerido en altas concentraciones puede ocasionar fluorosis dental, fluorosis ósea y otros efectos adversos.
La fluorosis dental inicia en la etapa de odontegénesis, y se caracteriza por la presencia de manchas blancas, opacas o amarillas. La fluorosis ósea genera deformaciones severas, así como la pérdida de movilidad en las extremidades. Estos efectos se dan como resultado de la mineralización del tejido óseo, reduciendo así la calidad del hueso (NRC, 2006).

En aguas subterráneas tanto someras como profundas se presenta la disolución de la fluorita, apatita u otro tipo de roca que contiene este mineral de origen volcánico. Pero de manera más general, la concentración de flúor en los acuíferos se ve incrementada conforme aumenta la profundidad del mismo (Ayoop y Gupta, 2006). Por esto es común encontrar este elemento en grandes cantidades en acuíferos sobreexplotados de las zonas desérticas.

Ayoop y Gupta (2006) estiman que aproximadamente 200 millones de personas en 25 naciones reconocidas por la UNICEF están destinadas a presentar fluorosis. China e India son los países con mayor problema de fluorosis, con un estimado de 60 millones de afectados entre ambas naciones. En el caso de México, la alta producción de fluorita y su origen volcánico, hacen evidente la presencia de flúor en diversas regiones del país.

Ante situaciones como ésta, la adecuada utilización del agua es necesario aplicar técnicas especiales para su purificación, de manera que pueda ser empleada para el consumo humano sin riesgos a la salud.

La remoción de sustancias disueltas del agua tales como el arsénico y el flúor requiere de métodos más complejos que las tecnologías convencionales de potabilización (coagulaciónsedimentación-filtración). Entre ellas se cuentan la ósmosis inversa, intercambio iónico, alúmina activada, nanofiltración y electrodiálisis inversa. Las tecnologías emergentes son: arena recubierta con óxidos de hierro, hidróxido férrico granular, empaques de hierro, hierro modificado con azufre, filtración con zeolita, etc. (Castro de Esparza, 2004).

Tomando en cuenta que estas tecnologías tienen como fundamento el uso de energías convencionales, se propone el método de destilación solar como una alternativa ideal para la desalinización de agua por su costo energético nulo y escasa inversión. 


\section{Justificación}

El estado de Chihuahua posee una amplia extensión de zonas áridas, por lo que el agua subterránea es el principal recurso para el abastecimiento público, así como para usos industriales y el riego agrícola. De los 61 acuíferos existentes, 30 presentan condiciones de sobreexplotación. Entre éstos se encuentra el acuífero Meoqui-Delicias (DOF, 2018).

La sobreexplotación del agua subterránea ocasionada por el desequilibrio entre la extracción y la recarga en los acuíferos impacta negativamente no sólo en el volumen de agua disponible, sino también en el deterioro de la calidad del agua, especialmente la que es destinada para el consumo humano.

Barrera (2008) realizó una investigación sobre calidad en las fuentes de agua potable ubicadas en la sección norte del acuífero Meoqui-Delicias, abarcando los municipios de Julimes, Rosales, Meoqui y Delicias. En dicho estudio se detectaron un gran número de poblaciones en las que el arsénico supera el valor límite de $0.025 \mathrm{mg} / \mathrm{l}$ establecido en la norma mexicana de agua potable ((Modificación a la NOM-127), siendo uno de los más afectados el municipio de Julimes. En dichas fuentes, además de las altas concentraciones de arsénico se observaron valores elevados de salinidad en términos de sólidos disueltos totales a causa de sodio, sulfatos, cloruros, así como también de fluoruros (Espino et al., 2009).

Con la finalidad de prevenir efectos adversos a la salud, la autoridad estatal ha gestionado la instalación de sistemas de ósmosis inversa para la desmineralización del agua que se destina al consumo directo en las localidades más pobladas. De esta manera ha quedado resuelto el problema de ingesta de agua con constituyentes adversos a la salud para la mayor parte de los habitantes del municipio.

No obstante lo anterior, existen pequeñas comunidades cuya localización remota dentro del municipio les dificulta el acceso a las plantas de tratamiento $\mathrm{y}$, por lo tanto, utilizan para consumo el agua de sus norias o pozos particulares. En estos pequeños poblados o rancherías se requiere contar con un proceso de eliminación de arsénico, flúor y otros elementos indeseables en el agua de consumo que sea económico, eficiente y de fácil operación.
Entre las diversas tecnologías existentes para la desalinización del agua, la destilación solar se presenta como un método ideal por su costo energético nulo y escasa inversión, basado en la utilización de una fuente energética renovable que no causa alteraciones al medio ambiente.

Esto es aplicable especialmente en zonas áridas que cuentan con altas tasas de radiación solar y que se encuentran aisladas de las localidades más pobladas.

Debido a su localización geográfica, gran parte del territorio chihuahuense cuenta con una tasa de radiación solar promedio de 6 $\mathrm{KW} / \mathrm{m}^{2}$ que viene siendo una de las más altas a nivel mundial (CNA, 2011).

\section{Hipótesis}

La destilación solar mejora la calidad del agua subterránea con altas concentraciones de arsénico y flúor hasta el grado del cumplimiento con el límite establecido para dicho elemento en la normatividad mexicana para agua potable

\section{Objetivo}

Operar un sistema de destilación solar para el tratamiento de agua subterránea caracterizada por su alto contenido de arsénico y flúor, con el fin de reducir estos parámetros a niveles de cumplimiento de la norma mexicana para consumo humano.

\section{Marco Teórico}

La radiación solar es el conjunto de emisiones de energía procedentes del núcleo del sol y que se transmiten por ondas electromagnéticas. La mayor radiación incidente sobre el planeta Tierra procedente del sol se estima que es, dependiendo del lugar y la época del año, de $1000 \mathrm{~W} / \mathrm{m}^{2}$ (Duffie y Beckman, 2013).

La energía emitida por el sol, cuando atraviesa la atmósfera de la Tierra, origina la evaporación del agua líquida causando la circulación atmosférica por la alteración en la densidad de los gases. Esta energía produce la temperatura en la superficie terrestre y es el motor del ciclo hidrológico. 
La destilación solar funciona mediante un sistema eficiente y sencillo, que permite reproducir de manera acelerada y controlada los procesos naturales de evaporación y condensación del agua. De acuerdo con Zargistalukder et al. (2013), en el proceso de destilación solar se replican las etapas del ciclo hidrológico.

Los principios de la destilación solar pueden ser aplicados a distintas escalas, desde pequeños destiladores, hasta grandes instalaciones. Esta tecnología se puede utilizar para eliminar eficazmente muchas impurezas que van desde sales hasta microorganismos, e incluso se emplea para obtener agua potable a partir de agua de mar (Zargistalukder et al., 2013).

Las regiones más favorables para los procesos de destilación solar se ubican entre las latitudes $15^{\circ} \mathrm{N}$ y $35^{\circ} \mathrm{N}$ (así como $15^{\circ} \mathrm{S}$ y $35^{\circ} \mathrm{S}$ ). Estas regiones semiáridas se caracterizan por la mayor cantidad de radiación solar, en donde más del $90 \%$ de la luz solar llega como radiación directa. En ellas la precipitación es escasa y no existe nubosidad abundante, por lo que se llegan a presentar más de 3000 horas de luz solar por año (Wegelin \& Meierhofer, 2003).

Está demostrado que el Estado de Chihuahua y en general el Norte de México recibe una incidencia de solar de las mayores que se tienen registradas a nivel mundial. Se reporta por especialistas en la materia, que en un día soleado normal, en esta región se reciben del orden de 4 a $6 \mathrm{Kw}$-h por metro cuadrado de energía de parte del sol (FRAPER, 2009).

\section{Metodología de la investigación}

\section{a) Descripción del destilador}

El destilador solar de doble vertiente utilizado en esta investigación consta de una estructura cuadrada, la cual se encuentra cubierta por un panel transparente dispuesto a dos aguas (Figura 1). La base de la estructura es un contenedor de fondo negro, donde se coloca el agua a destilar. El panel superior transparente permite el paso de la radiación solar y a su vez mantiene cerrada la estructura. Mediante el calor generado por la radiación el agua se evapora y asciende por la estructura mediante un proceso de convección.
El vapor entra en contacto con la superficie transparente que se encuentra a una temperatura menor que el fondo del destilador, por lo que se condensa en forma de gotas. Finalmente, éstas se deslizan por el panel hacia las orillas donde son colectadas en un recipiente colocado debajo de la bandeja alimentadora (Figura 2).

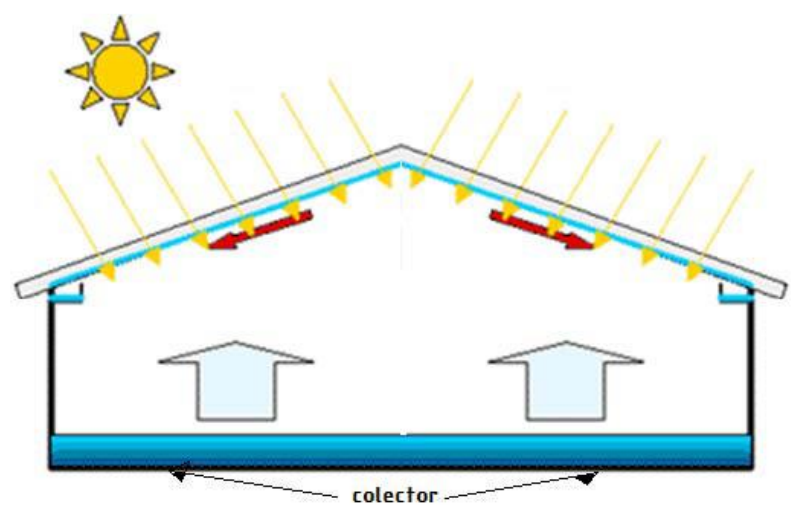

Figura 1 Esquema del destilador de doble vertiente

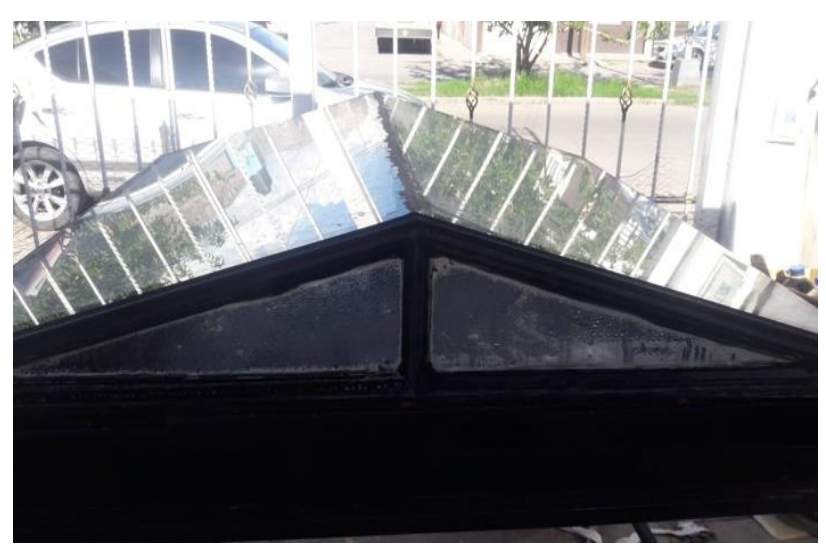

Figura 2 Destilador solar ubicado en el sitio de estudio

b) Procedimiento experimental

Alimentación del destilador

El destilador solar se alimentó en etapas sucesivas con el agua procedente de pozos de las comunidades La Regina (LR), San José (SJ) y La Esperanza (LE) del municipio de Julimes, Chih., caracterizados por la presencia de altas concentraciones de arsénico (As) y flúor $(\mathrm{F})$.

Para un correcto control del afluente o agua de ingreso en el destilador, en la charola se alimentaron diariamente 3 litros de agua con alto contenido de arsénico y flúor. La alimentación se realizó al final de cada período diurno del experimento, a fin de asegurar la mayor captación de radiación solar al inicio del día siguiente. 


\section{Recolección del destilado}

La recolección diaria del destilado se llevó a cabo al finalizar el período de iluminación, con la finalidad obtener la mayor cantidad de condensado una vez que el destilador hubiera alcanzado la temperatura ambiente.

\section{Análisis de muestras}

Después de colectar el destilado se analizó en cada muestra la conductividad eléctrica (CE) y sólidos disueltos totales (SDT) empleando para ello un equipo portátil Sension 5 marca HACH. El resto del destilado se almacenó en el frigorífico para análisis posteriores.

Contando con información de las condiciones climatológicas registrada por la Comisión Nacional del Agua para la zona, durante los períodos de experimentación para los 3 tipos de agua se registraron los siguientes datos ambientales: temperatura ambiental máxima y mínima, velocidad y dirección del viento, porcentaje de humedad y nubosidad, así como precipitación pluvial diaria.

Con el apoyo de un sensor eléctrico equipado con termopares (Figura 3) se registraron también lecturas horarias de temperatura ambiente (exterior), del vapor y del agua dentro del destilador, correspondientes a un período diario de experimentación para cada una de las fuentes de agua estudiadas.

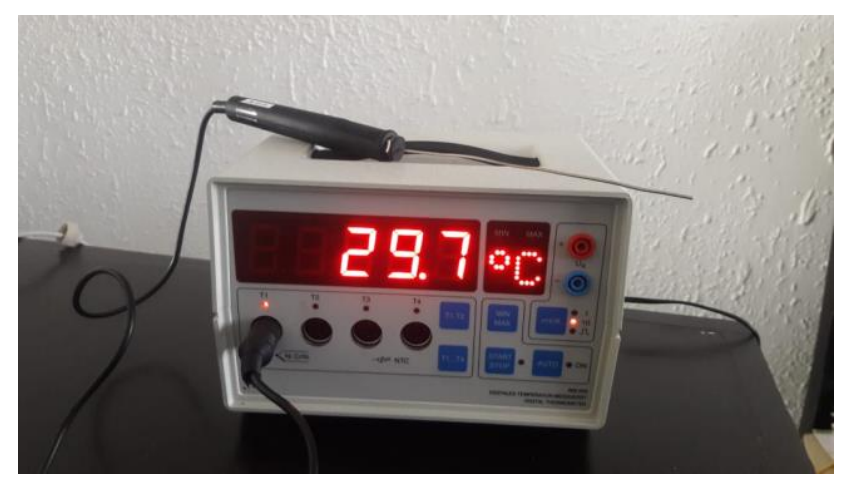

Figura 3 Sensor de temperatura a base de termopares

\section{Resultados}

Condiciones ambientales

Los resultados de destilación de agua de la fuente La Regina que se reportan corresponden a los días 9, 13 y 14 de agosto de 2018.
Para fines prácticos se dio a cada muestra colectada un código de identificación (D1, D2 y D3, respectivamente). Durante este período de experimentación el volumen promedio colectado fue de $1.8 \mathrm{~L} / \mathrm{d}$.

Con el agua de la fuente San José se trabajó en el destilador durante 6 días, de manera que el código de identificación de las muestras colectadas diariamente inicia con D1 y concluye con D6; estas muestras corresponden respectivamente a las fechas $16,17,18$ y 19 de agosto, así como 9 y 10 de septiembre de 2018 . En estos días de experimentación el volumen de destilado promedio colectado fue de $1.4 \mathrm{~L} / \mathrm{d}$.

Los resultados de destilación del agua de la fuente La Esperanza se obtuvieron durante 4 días $(25,26,28$ y 29 de agosto de 2018) identificados con los códigos D1, D2, D3 y D4, respectivamente. Durante este periodo el volumen promedio de destilado colectado fue de $1.2 \mathrm{~L} / \mathrm{d}$.

En la Tabla 1 se muestra un resumen de los valores de temperatura ambiental máxima y mínima, así como de las condiciones climatológicas imperantes durante los períodos de experimentación. Dichas condiciones son velocidad y dirección del viento, porcentaje de humedad relativa, nubosidad y precipitación pluvial. Esta información fue obtenida en la página web del Servicio Meteorológico Nacional de la Comisión Nacional del Agua (CONAGUA, 2018).

\begin{tabular}{|lccc|}
\hline \multicolumn{4}{c|}{ FUENTE DE AGUA } \\
\hline & LA REGINA & SAN JOSÉ & LA ESPERANZA \\
\hline Núm. de mediciones & 3 & 6 & 4 \\
Temperatura máx. $\left({ }^{\circ} \mathrm{C}\right)$ & 32.9 & 32.9 & 33.4 \\
Temperatura mín. $\left({ }^{\circ} \mathrm{C}\right)$ & 24.9 & 23.6 & 24.6 \\
Vel. del viento(km/h) & 10.0 & 5.2 & 7.0 \\
Dirección del viento & ESTE & NORESTE & NORESTE \\
Humedad (\%) & 63.3 & 43.5 & 33.3 \\
Nubosidad $(\%)$ & 52.3 & 30.3 & 12.5 \\
Precipitación (mm) & 0.4 & 0.1 & 0.0 \\
Volumen colectado(L/d) & 1.8 & 1.4 & 1.2 \\
\hline
\end{tabular}

Tabla 1 Información climatológica (CNA, 2018) 
En las Figuras 4, 5 y 6 se muestran las mediciones horarias de temperatura ambiente (exterior), así como del vapor y del agua dentro del destilador, correspondientes a un período diario de experimentación para cada una de las fuentes de agua estudiadas. Se observa que, a pesar de las temperaturas exteriores máximas detectadas variando entre 33 y $36{ }^{\circ} \mathrm{C}$, dentro del destilador en las horas pico de insolación se alcanzaron temperaturas entre 66 y $68{ }^{\circ} \mathrm{C}$ para el vapor y en el rango de 70 a $74{ }^{\circ} \mathrm{C}$ en el agua durante el proceso de destilación.

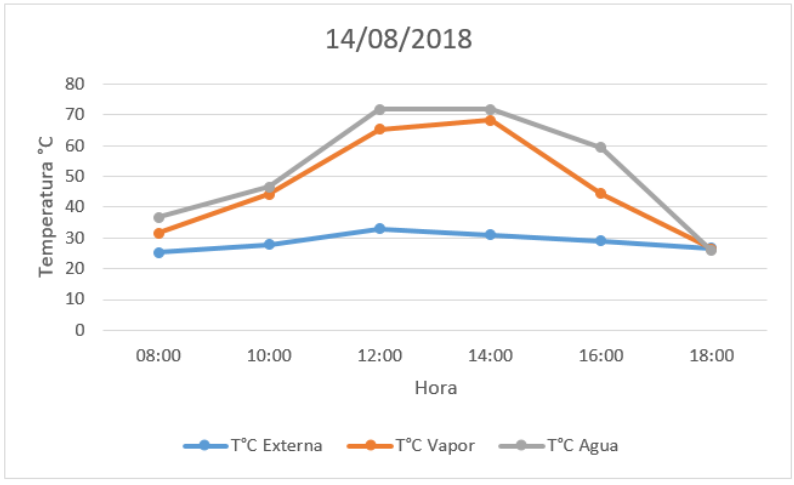

Figura 4 Variación de temperatura en el destilador el día 14/08/2018 (D3). Fuente de agua: La Regina

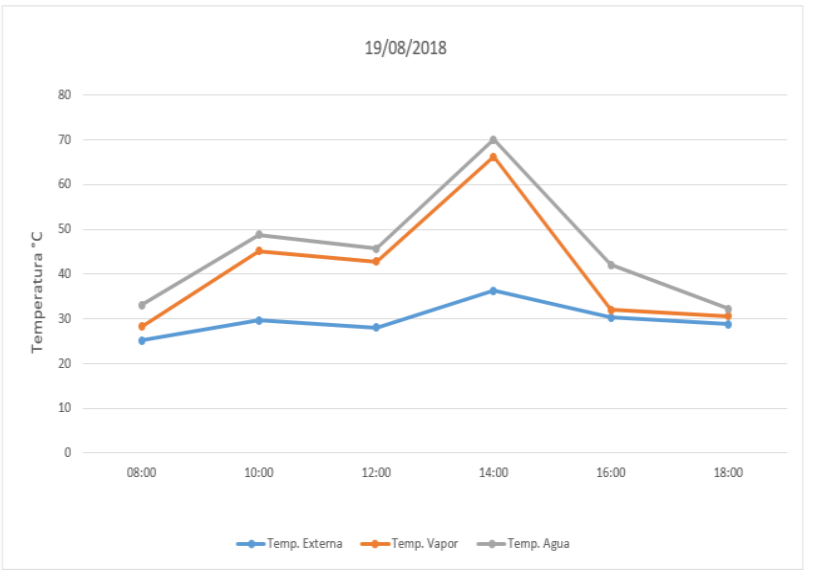

Figura 5 Variación de temperatura en el destilador el día 19/08/2018 (D4). Fuente de agua: San José

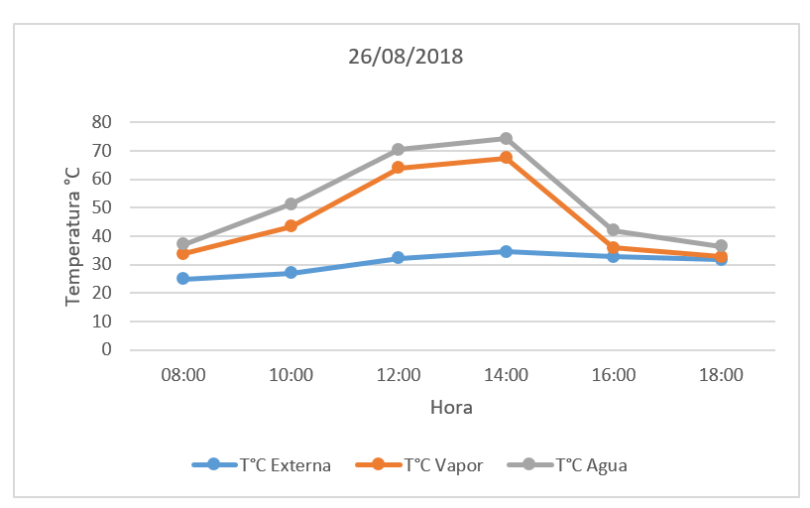

Figura 6 Variación de temperatura en el destilador el día 26/08/2018 (D2). Fuente de agua: La Esperanza

\section{Resultados de calidad de agua}

\section{Conductividad Eléctrica}

La Conductividad Eléctrica es una medida de de la capacidad del agua para transportar la corriente eléctrica, por lo que está relacionada directamente con la concentración de iones o sales disueltas que están presentes en la misma. En las Figura 7 se aprecian las variaciones de este parámetro entre el agua del pozo y los destilados de las fuentes La Regina (a), San José (b) y La Esperanza (c), cuyos valores antes del tratamiento son de 2570, 2140 y $1630 \mu \mathrm{S} / \mathrm{cm}$, respectivamente, y que corresponden a 1800 , 1500 y $1140 \mathrm{mg} / \mathrm{l}$ en términos de sólídos disueltos totales (SDT); cabe mencionar que estos valores superan el límite máximo permisible de $1000 \mathrm{mg} / \mathrm{l}$ establecido en la normatividad mexicana para agua potable (Modificación a la NOM-127) para dicho parámetro.

En dicha figura puede observarse que los resultados después del proceso de destilación solar indican disminuciones de conductividad eléctrica entre 98.5 y $99 \%$, con valores promedio respectivos de $21,16.8$ y $24 \mu \mathrm{S} / \mathrm{cm}$ para las tres fuentes de agua estudiadas.

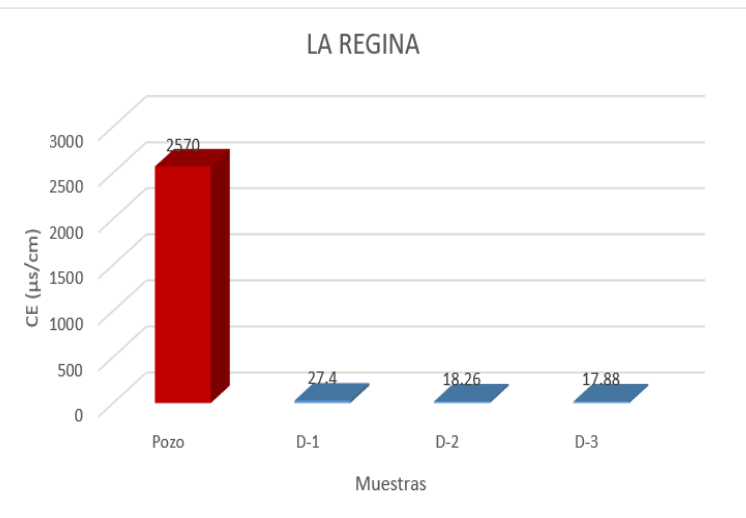

(a)

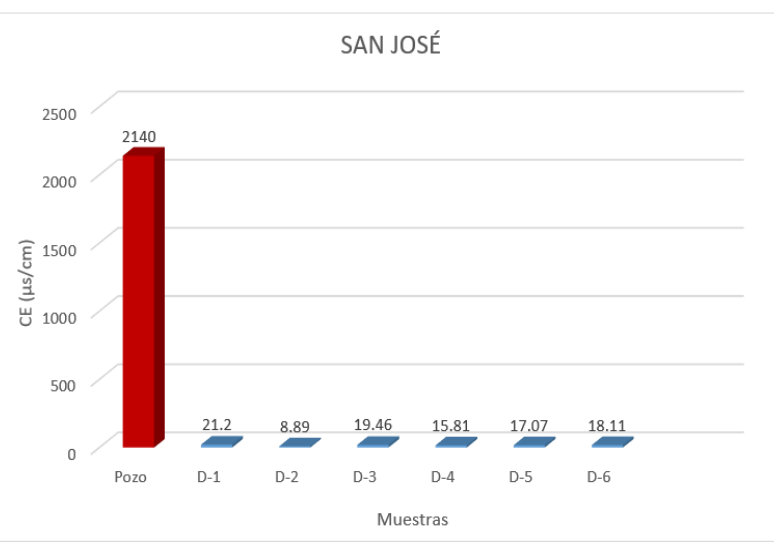

(b)

ESPINO-VALDÉS, María Socorro, HERNÁNDEZ-HERRERA, Cristina, PINALES-MUNGUÍA, Adán y BALLINAS-CASARRUBIAS, María de Lourdes. Utilización de un sistema de destilación solar para remoción de Arsénico y Flúor en agua subterránea destinada al consumo humano. Revista de Ingeniería Biomédica y Biotecnología. 2019 


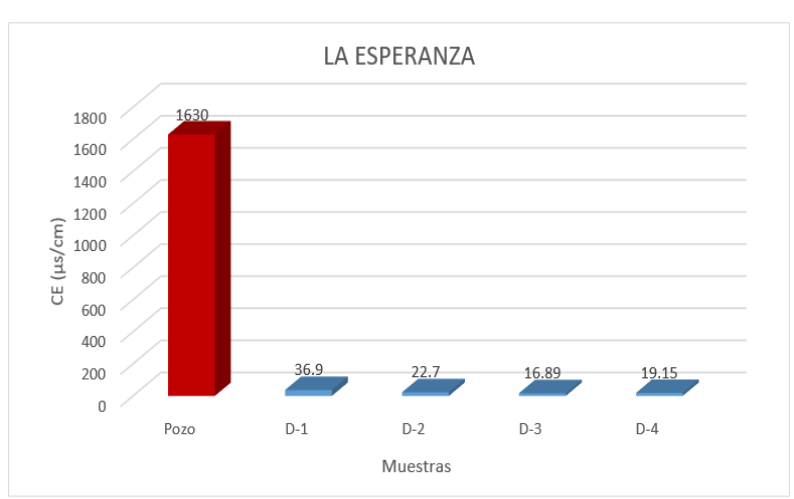

(c)

Figura 7 Resultados de remoción de Conductividad Eléctrica en el destilador operado con agua de La Regina (a), San José (b) y La Esperanza (c)

\section{Arsénico}

Los resultados del proceso de destilación en lo que a arsénico se refiere se muestran en las Figura 8. En ellas se aprecia que las concentraciones de este elemento en las fuentes de agua de La Regina (a), San José (b) y La Esperanza (c) antes del tratamiento fueron de $0.145 \mathrm{mg} / \mathrm{L}, \quad 0.194 \quad \mathrm{mg} / \mathrm{L}$ y $0.136 \mathrm{mg} / \mathrm{L}$ respectivamente, mismas que superan el límite de $0.025 \mathrm{mg} / \mathrm{L}$ establecido para el agua potable. Los resultados de arsénico reportados para las muestras de destilado de los 3 pozos fueron ND (No Detectado) en relación al límite de detección $(0.001 \mathrm{mg} / \mathrm{L})$ del equipo de absorción atómica utilizado para la medición, con lo que se concluye una eficiencia de $100 \%$ en la remoción de dicho elemento .

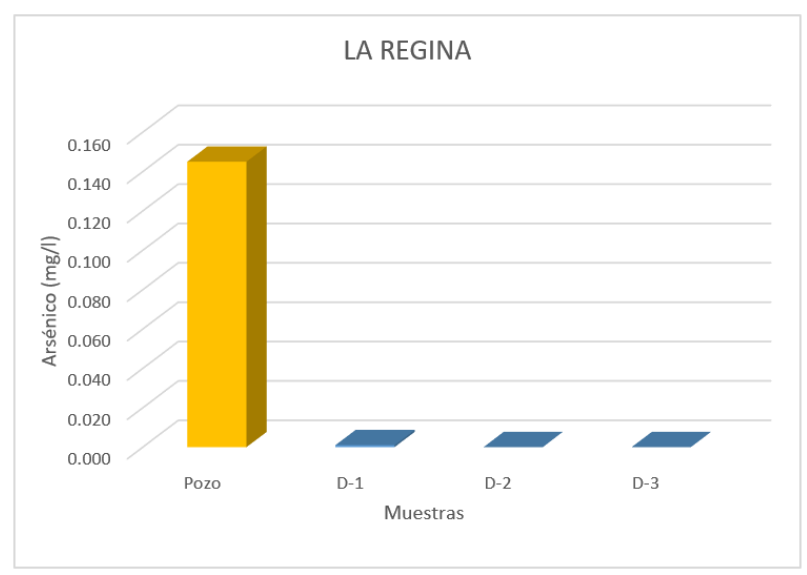

(a)

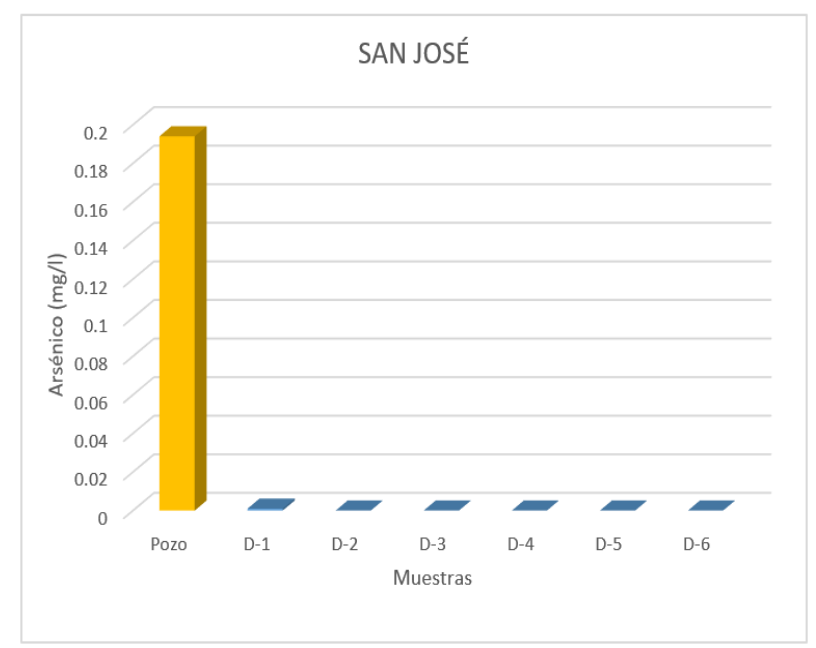

(b)

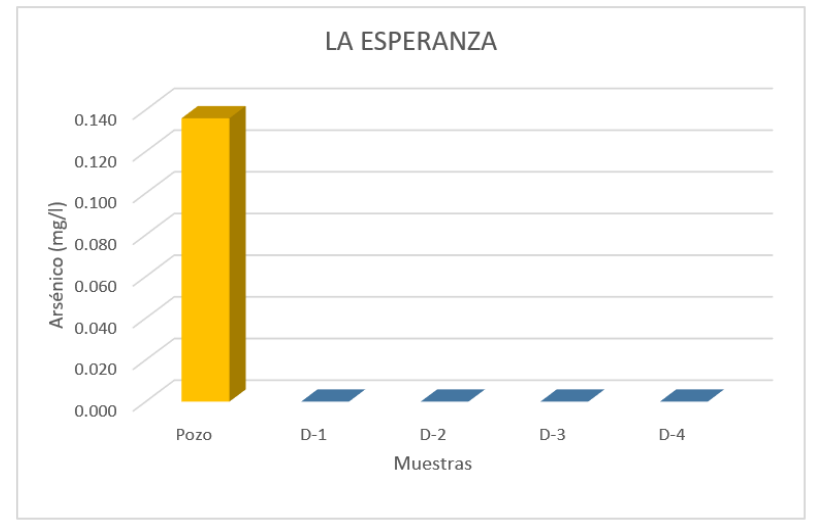

(c)

Figura 8 Resultados de remoción Arsénico en el destilador operado con agua de La Regina (a), San José (b) y La Esperanza (c)

\section{Flúor}

En las Figura 9 se aprecian las variaciones del flúor antes y después del proceso de destilación correspondientes a los pozos de La Regina (a), San José (b) y La Esperanza (c), cuyos valores antes del tratamiento fueron 5.26, 7.42 y 4.49 $\mathrm{mg} / \mathrm{L}$, respectivamente; todos ellos por encima del límite máximo permisible de $1.5 \mathrm{mg} / \mathrm{l}$ establecido en la normatividad mexicana para agua potable para dicho parámetro.

Los resultados después del proceso de destilación solar indican disminuciones muy significativas de flúor hasta valores promedio de $0.18,0.13$ y $0.16 \mathrm{mg} / \mathrm{L}$, respectivamente. $\mathrm{La}$ comparación de dichos valores con las concentraciones originales indican eficiencias entre $96.4 \%$ y $99 \%$ para la remoción de flúor. 


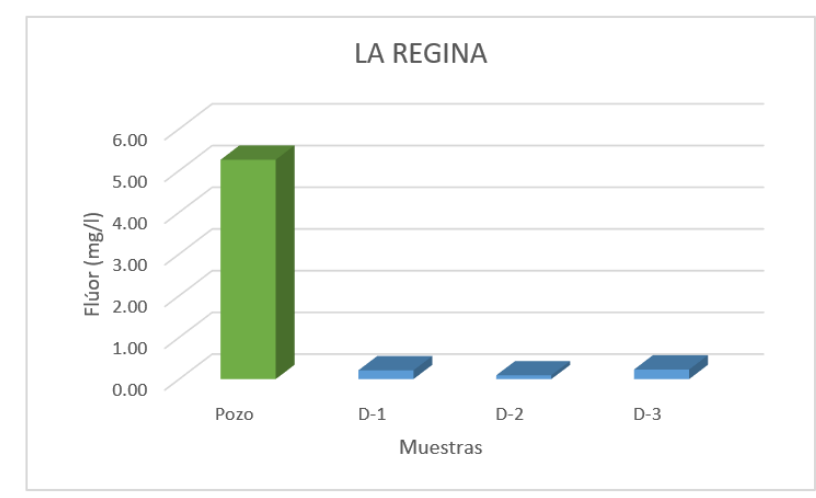

(a)

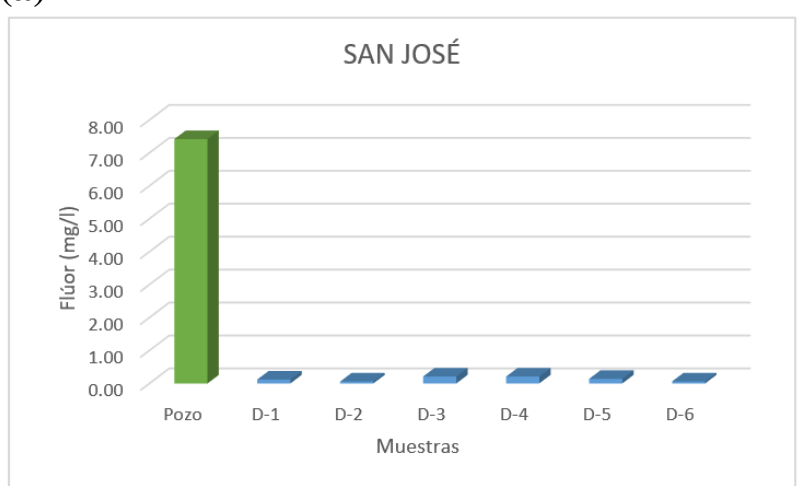

(b)

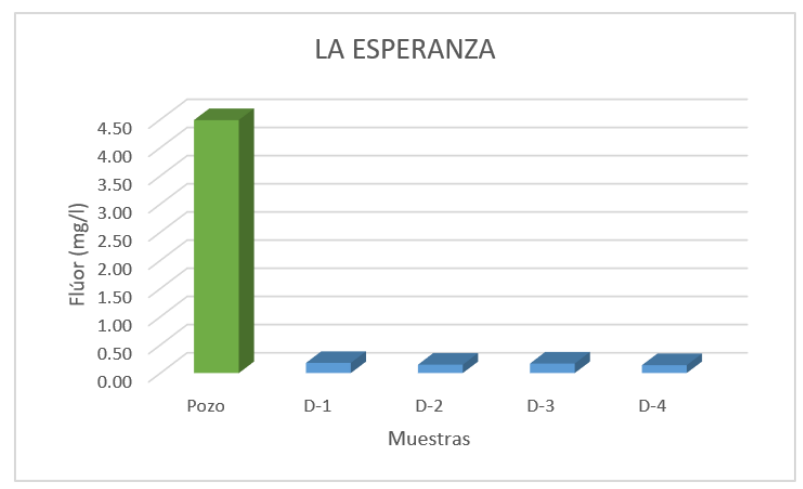

(c)

Figura 9 Resultados de remoción de Flúor en el destilador operado con agua de La Regina (a), San José (b) y La Esperanza (c)

\section{Conclusiones}

- Mediante el sistema experimental de destilación solar fue posible disminuir las altas concentraciones de arsénico y flúor presentes en tres fuentes de aguas subterránea hasta valores muy por debajo de los límites establecidos en la norma oficial mexicana para agua potable.

- $\quad$ El arsénico en los tres tipos de agua empleados fue removido con una eficiencia de $100 \%$.

- $\quad$ El flúor fue removido en el sistema con eficiencias entre 96 y $99 \%$, dando como resultado destilados con concentraciones entre 0.13 y $0.18 \mathrm{mg} / \mathrm{L}$.
- $\quad$ La salinidad del agua de las tres fuentes en términos de conductividad eléctrica se logró disminuir entre 98 y $99 \%$, dando como resultado destilados con menos 25 $\mu \mathrm{S} / \mathrm{cm}$.

- $\quad$ La calidad del agua obtenida mediante el sistema probado permite que ésta sea empleada para el consumo humano sin que existan posibles riesgos a la salud de los consumidores por presencia de arsénico y flúor.

\section{Referencias}

Ayoop, S. y A. K. Gupta. (2006)._Fluoride in drinking water: a review on the status and stress effects. Critical Reviews in Environmental Science and Technology; 2006, Vol. 36, pp 433487.

Barrera, Y. (2008). Estudio hidrogeoquímico y de vulnerabilidad a la contaminación del acuífero Meoqui- Delicias. Tesis de Maestría, Facultad de Ingeniería, Universidad Autónoma de Chihuahua.

Bocanegra, O. C., Bocanegra, E. M., \& Alvarez, A. A. (2002). Arsénico en las aguas subterráneas: su impacto en la salud. In: Proceedings of the Congreso Internacional XXXII IAH y VI ALHSUD, Aguas Subterráneas y Desarrollo Humano (Vol. 21, pp. 21-2).

Castro de Esparza, M. L. (2006). Presencia de arsénico en el agua de bebida en América Latina y su efecto en la salud pública. In International Congress Natural Arsenic in Groundwater in Latin America.

Castro de Esparza, M. L. (2004). Remoción del arsénico en el agua para bebida $\mathrm{y}$ biorremediación de suelos. In International Congress Natural Arsenic in groundwaters of Latin America.

CONAGUA (2007). Diseño de plantas potabilizadoras tipo de tecnología simplificada. Manual de Agua Potable, Alcantarillado y Saneamiento. Julio 2007.

CONAGUA (2011). Estadísticas del Agua en México, Edición 2011. Cap. 8. Agua en el mundo. 
CONAGUA (2018). Comisión Nacional del Agua. Servicio Meteorológico Nacional. Página web https://smn.cna.gob.mx

DOF (2018). Diario Oficial de la Federación. Acuerdo por el cual se actualiza la disponibilidad de agua subterránea de los 653 acuíferos de los Estados Unidos Mexicanos, mismos que forman parte de las Regiones Hidrológico-Administrativas que se indican. 04 de enero de 2018.

Duffie, J. y Beckman, W. (2013). Solar Energy Thermal Processes, Fourth Edition. Solar Energy Laboratory. University of WisconsinMadison. Wiley.

EPA (2016). Environmental Protection Agency.Arsenic compounds. https://www.epa.gov/sites/production/files/2016 -09/documents/arsenic-compounds.pdf

Espino, M. S., Barrera, Y., \& Herrera, E. (2009). Presencia de arsénico en la sección norte del acuífero Meoqui-Delicias del estado de Chihuahua, México. TECNOCIENCIA, Vol. II, No.1.

FRAPER. 2009. Foro Regional de Análisis del Potencial Energético Renovable. Potencial de Energías Renovables en Chihuahua. Cd. Juárez, Chih. 8 y 9 de diciembre de 2009.

Modificación a la Norma Oficial Mexicana NOM-127-SSA1-1994. (2000). Salud Ambiental. Agua para Uso y Consumo Humano. Límite Permisibles de Calidad y Tratamientos a que debe someterse para su potabilización. Secretaría de Salud. México, D.F.

NRC (2006). National Research Council. Committee on Fluoride in Drinking Water, Fluoride in Drinking Water: A Scientific Review of EPA's Standards. National Research Council. National Academic of Sciences, March 2006. 500 fifth Street NW, Washington CD 20001, USA. http://www.nap.edu/catalog/11571.html

Wegelin, M. y R. Meierhofer. 2003. Desinfección Solar del Agua. Guía de aplicación. Fundación SODIS para América Latina. Lima, Peru: Indeart, 2003.
Zargistalukder, M., A. Foisal, A Siddique \& M. Rafiqulalambeg (2013). Design and Performance of Solar Water Distillation. Global Jorunal or Resarches in Engineering. Vol. 13 Issue 1 Version 1.0. 2013. 